\title{
The experience of psychiatrists managing Chinese patients in Merseyside
}

\author{
D. LI, Senior Registrar in Psychiatry, Winwick Hospital, Winwick, \\ Warrington WA2 8RR
}

The Chinese community in the UK has existed since the latter half of the 1800 s and traditionally has been perceived as insular and self sufficient (Lynn, 1982). In the 1960s and '70s, there was a large influx of Chinese into the UK to work in catering businesses. Many of the children of this generation were born in this country; with this group now in their early adulthood, there is more integration into the indigenous population. However, there is still an impression that Chinese psychiatric patients are under-represented in the services, but as yet, there has been little psychiatric research carried out among the Chinese in the UK. This study examined briefly the level of contact with, and difficulties encountered in, managing Chinese patients by psychiatrists in Merseyside.

\section{The study}

All consultants and senior registrars (including those in honorary posts) in the Mersey region were sent a questionnaire, which was completed and returned over a two-month period between June and August 1990.

The questionnaire contained 12 items which addressed the following areas:

(1) The subspeciality of the respondent.

(2) The number of Chinese patients under the respondent's care and whether this was considered to be more/same/less than would be expected.

(3) Do Chinese patients pose greater management problems? If so, whether these are due to cultural/language/social difficulties,

(4) Do language difficulties prevent a significant number of Chinese patients from using the services?

(5) Is interviewing through a non-medical interpreter generally satisfactory? If so, whether they are easy to find.

(6) Is there sufficient information available about the Chinese culture?

(7) Would there be a place for a team familiar with the Chinese culture to manage Chinese psychiatric patients?

\section{Findings}

One hundred and twenty-two questionnaires were sent out: there were 93 replies (76\% response rate) with five undelivered. Of these, 72 were consultant replies: adult general/forensic/alcohol and substance abuse psychiatry $(n=43)$; psychogeriatrics $(n=11)$; and child/mental handicap psychiatry $(n=18)$.

Of the consultants, $29 \%$ had Chinese patients under their care at the time of the survey; among the adult psychiatrist's group, $40 \%$ had Chinese patients but only two consultants had more than five patients. Most $(72 \%)$ thought that this under-represented the actual numbers (none considered the numbers to be an over-representation).

With consultants and senior registrars considered together, $47 \%$ thought that Chinese patients posed greater management difficulties (compared to $26 \%$ who disagreed) but under a half of these thought that the difficulties were actually major. If only those consultants with Chinese patients under their care were considered (total number was 21 ), the $47 \%$ rose to $62 \%$. A large number $(49 \%)$ made no reply when asked what they considered the difficulties may be due to, many commenting that they had had insufficient experience. Of those who did reply, some twothirds (which includes $54 \%$ of those with Chinese patients in their care), thought the difficulties were due to language problems in contrast to the remaining third (12\% of those with Chinese patients) who considered cultural/environmental factors to be more important. Forty-three per cent thought language difficulties prevented service utilisation in a significant number, with $14 \%$ disagreeing. Again, when only those with Chinese patients were considered, $57 \%$ thought it did cause problems in using psychiatric facilities. Interpreters were difficult to find for $57 \%$ of those who actually had Chinese patients, and $67 \%$ of them said that the service provided by interpreters was generally unsatisfactory. The majority of those replying ( $46 \%$, with $47 \%$ making no reply), thought there was insufficient information on the Chinese culture. Of those replying when asked about the feasibility of a 'Chinese team', about similar numbers were for and against such an idea, but several commented on the need for a 
community psychiatric nurse working either full- or part-time with Chinese patients.

At the time of the questionnaire, four of the 18 consultants in child psychiatry or mental handicap and none of the psychogeriatricians had Chinese patients in their care. In general, the results were similar for the different subspecialty groups but there were a few differences. The psychogeriatricians considered Chinese patients to pose greater problems in management (yes:no ratio of $55 \%: 9 \%$ ), whereas the results for the adult psychiatry and the child/mental handicap psychiatry groups were less clear (42\%: $35 \%$ and $39 \%: 28 \%$ respectively). When those with Chinese patients currently under their care were considered, the figures for the latter two groups changed to $65 \%: 35 \%$ and $50 \%: 50 \%$ respectively. Within the child/mental handicap psychiatry group, $33 \%$ considered non-medical interpreters to be satisfactory and $23 \%$ thought they were unsatisfactory. For the other two groups, the differences were unequivocal (47\%: $17 \%$ and $64 \%: 9 \%$ ).

\section{Comment}

The study was a brief, non-standardised survey which has obvious limitations. However, the response of $76 \%$ was good and is likely to be a fairly accurate reflection of the experience of managing Chinese patients in the Mersey region, from the perspective of psychiatrists. Whether these findings are applicable to other regions is not known.

Overall, $29 \%$ had care of Chinese patients but the numbers were low; in addition many questions were not answered as the respondents considered themselves to have had insufficient experience of Chinese patients. This is consistent with reports (Chan, 1986) that found a tendency for the greater part of the Chinese community in the UK to be scattered. Chinatowns and their surrounding areas may have a significant number of Chinese residents but they are primarily foci for social activities and employment. In the Mersey region, there is a relative concentration of Chinese people in the south-central catchment areas of Liverpool. The majority of the respondents expected more Chinese psychiatric patients from their estimate of the size of the population in their areas. Whether this is due to a lower prevalence of psychiatric morbidity or an under-utilisation of the services, is not clear. A recent study of Chinese primary care patients in Manchester (Pan \& Goldberg, 1990) suggests the latter but further fundamental research needs to be done. The results of the 1991 census should be available in the next few years, providing accurate demographic data for the different ethnic groups and this should facilitate the task.

Language difficulties were perceived to be more important than cultural/environmental factors in leading to management problems and service underutilisation; interpreters do not appear to be a satisfactory solution to this. These trends were more apparent with those who had Chinese patients under their care; presumably, their opinion approximates better to the actual situation. Whether the use of interpreters with basic psychiatric knowledge is the answer no-one knows, but there remains the problems of the difficulties encountered in using the existing services. Many thought information on Chinese culture was lacking and the production of an information pack may be a simple initial step in addressing this issue.

In the new world of 'purchasers' and 'providers', it is expected that ethnic groups will be one of the areas monitored in respect to the delivery and uptake of services. The employment of community nurses fluent in Chinese and familiar with the culture would be a viable and effective first step to overcoming some of the problems encountered in managing Chinese psychiatric patients. Community nurses would be effective in dealing with the scattered nature of the population and their role would include: aiding accurate diagnosis, monitoring and working with the patient in the context of the family.

\section{Acknowledgements}

My thanks to all the psychiatrists in Mersey region who completed the questionnaire. I wish to thank Dr I. A. Davidson for his helpful comments during the preparation of the questionnaire and in the writing of this report, and also, thanks to Karen Li for help in typing and correcting my grammar.

\section{References}

Chan, A. (1986) Employment Prospects of Chinese Youth in Britain: a research report, July 1986. London: Commission for Racial Equality.

LYNN, I. L. (1982) The Chinese Community in Liverpool: their unmet needs with respect to education, social welfare and housing. University of Liverpool: Merseyside Area Profile Group.

PAN , P. C. \& Goldberg, D. (1990) A comparison of the validity of GHQ-12 and CHQ-12 in Chinese primary care patients in Manchester. Psychological Medicine, 20, 931-940. 